03

\title{
Моделирование и расчет течений наножидкости в пограничном слое
}

\author{
(c) T.P. Аманбаев
}

Южно-Казахстанский государственный университет им. М. Ауэзова, 160012 Шымкент, Казахстан

Институт математики и математического моделирования, 050010 Алматы, Казахстан

e-mail: tulegen_amanbaev@mail.ru

(Поступило в Редакцию 17 ноября 2017 г.)

В рамках диффузионного приближения рассматривается течение так называемой наножидкости (дисперсной смеси жидкости с наноразмерными частицами) в пограничном слое. Анализируются случаи, приводящие к несжимаемости наножидкости. Сформулирована автомодельная задача, для решения которой используется численный метод. Обсуждены некоторые результаты расчетов, иллюстрирующие характер поведения наножидкости в пограничном слое на продольно обтекаемой пластине при разных температурных режимах.

DOI: 10.21883/JTF.2018.11.46623.2567

\section{Введение}

Рассмотрим течение суспензии жидкости с наноразмерными частицами, называемой наножидкостью [1]. Интерес к моделированию и изучению поведения наножидкостей (или наносуспензий) обусловлен их возможным широким практическим применением. С ростом производительности электронных устройств и развитием высокоэнергетических технологий возникает необходимость создания эффективных охлаждающих систем и управления большими тепловыми потоками [2]. Один из способов интенсификации теплообмена - повышение теплопроводности жидкости путем добавления твердых частиц с высокой теплопроводностью. Особый интерес при создании таких суспензий представляют наночастицы. В отличие от частиц микронного размера, они медленнее осаждаются, не приводят к засорению и износу каналов. В то же время наночастицы склонны к диффузии.

Моделирование и решение задачи о течении наножидкости при наличии диффузионных потоков представляет собой достаточно сложную проблему, поэтому обычно такие задачи рассматриваются при упрощающих допущениях (несжимаемость суспензии, малость объемной концентрации частиц, пренебрежение влиянием частиц на теплофизические параметры суспензии, приближение пограничного слоя и т. п.) [3-6].

Цель настоящей работы - моделирование и изучение поведения наножидкости в пограничном слое на плоской пластине при наличии диффузионных потоков. Процесс теплообмена между наножидкостью и стенкой происходит в узком пограничном слое вблизи стенки. В связи с этим исследование характеристик наносуспензии в пограничном слое является актуальным и имеет важное прикладное значение.

\section{1. Основные допущения и уравнения}

\section{1. Основные допущения}

Наножидкость (или наносуспензия) представляет собой смесь жидкой (несущей) и твердой (дисперсной) фаз. Полагается, что дисперсную фазу составляют сферические частицы. Каждая из фаз в отдельности несжимаема. Фазовые и химические превращения, а также внешние силы отсутствуют.

Далее параметры несущей и дисперсной фаз будут отмечаться индексами 1,2 соответственно, а параметры смеси в целом - без индекса.

Наряду с параметрами, определяющими состояние отдельных компонентов, такими как истинные (отмечены верхним знаком „о “) и приведенные плотности $\rho_{1}^{\circ}, \rho_{2}^{\circ}$, $\rho_{1}, \rho_{2}$, объемные доли $\alpha_{1}, \alpha_{2}$, скорости $\mathbf{v}_{1}, \mathbf{v}_{2}$, и т. п., в механике смесей вводятся параметры, характеризующие смесь в целом, а именно: плотность смеси $\rho$ и среднемассовая (барицентрическая) скорость смеси $\mathbf{v}[7,8]$

$$
\begin{gathered}
\rho=\rho_{1}+\rho_{2}, \quad \rho_{1}=\alpha_{1} \rho_{1}^{\circ}, \quad \rho_{2}=\alpha_{2} \rho_{2}^{\circ}, \\
\alpha_{1}+\alpha_{2}=1, \quad\left(\rho_{1}^{\circ}, \rho_{2}^{\circ}=\text { const }\right), \\
\rho \mathbf{v}=\rho_{1} \mathbf{v}_{1}+\rho_{2} \mathbf{v}_{2} .
\end{gathered}
$$

Заметим, что так определенная скорость $\mathbf{v}$ представляет собой скорость общего центра масс индивидуальных объемов, соответствующих разным компонентам смеси.

\section{2. Уравнения движения и теплообмена суспензии}

Запишем уравнения неразрывности для компонентов смеси при наличии процессов диффузии [7-9]

$$
\frac{\partial \rho_{m}}{\partial t}+\nabla \cdot \rho_{m} \mathbf{v}=-\nabla \cdot \mathbf{J}_{m}, \quad \mathbf{J}_{m}=\rho_{m}\left(\mathbf{v}_{m}-\mathbf{v}\right), \quad m=1,2
$$


Здесь $\mathbf{J}_{1}, \mathbf{J}_{2}-$ векторы потока диффузии. Разность скорости $\mathbf{v}_{m}-\mathbf{v}$ (которую иногда называют диффузионной скоростью) является, очевидно, скоростью $m$-го компонента относительно центра масс смеси в целом. Члены $\nabla \cdot \mathbf{J}_{\mathbf{m}}$ характеризуют изменение массы $m$-го компонента в объеме, движущемся со скоростью $\mathbf{v}$, за счет того, что этот объем, если $\mathbf{v}_{m} \neq \mathbf{v}$, не является индивидуальным объемом для $m$-го компонента смеси. Частицы, составляющие $m$-й компонент, входят в этот объем и выходят из него. Очевидно, имеет место соотношение $\mathbf{J}_{1}+\mathbf{J}_{2}=0$.

Отметим, что вместо двух уравнений неразрывности для компонентов смеси (2) можно использовать уравнение неразрывности для одного из компонентов (скажем, для дисперсного компонента) и уравнение неразрывности для смеси в целом, которое получается сложением друг с другом уравнений неразрывности для компонентов (2)

$$
\frac{\partial \rho}{\partial t}+\nabla \cdot \rho \mathbf{v}=0
$$

Относительные движения компонентов, описываемые диффузионными скоростями, непосредственно влияют лишь на концентрацию компонентов и определяются диффузионным механизмом. Законы диффузии устанавливают зависимость мгновенных значений диффузионных потоков от градиентов концентраций, температуры и т. п. Использование этих законов диффузии предполагает пренебрежение инерцией относительного движения компонентов смеси (так называемое диффузионное приближение) [8].

Уравнение сохранения количества движения смеси (или уравнение Навье-Стокса) в рамках диффузионного приближения запишем в проекциях на координатные оси (считается, что тензор скоростей деформации определяется полем барицентрических скоростей смеси) [9]

$$
\begin{aligned}
& \rho\left(\frac{\partial v_{i}}{\partial t}+v_{k} \frac{\partial v_{i}}{\partial x_{k}}\right)=-\frac{\partial p}{\partial x_{i}}+\frac{\partial}{\partial x_{k}} \\
& \times\left\lfloor\mu\left(\frac{\partial v_{i}}{\partial x_{k}}+\frac{\partial v_{k}}{\partial x_{i}}-\frac{2}{3} \delta_{i k} \frac{\partial v_{j}}{\partial x_{j}}\right)\right\rfloor+\frac{\partial}{\partial x_{i}}\left(\xi \frac{\partial v_{j}}{\partial x_{j}}\right) .
\end{aligned}
$$

Здесь $x_{i}, v_{i}$ - пространственные координаты и соответствующие компоненты вектора скорости, $p$ - давление, $\mu, \xi$ - эффективные вязкости смеси ( $\mu$ - динамическая вязкость, а $\xi$ часто называют второй вязкостью $[9,10])$, $\delta_{i k}$ - символ Кронекера, $i, j, k=1,2,3$ (по $j, k$ производится суммирование). В уравнении (3) величины $\mu$, $\xi$ могут зависеть, вообще говоря, от температуры $T$, объемной концентрации дисперсных частиц $\alpha_{2}$ и т.п. В общем случае $T, \alpha_{2}$, а потому и $\mu, \xi$ не постоянны во всем объеме жидкости, так что параметры $\mu, \xi$ не могут быть вынесены из-под знака производной.

В случае несжимаемой среды, когда $\nabla \cdot \mathbf{v}=0$, уравнение (3) примет более компактную форму

$$
\rho\left(\frac{\partial v_{i}}{\partial t}+v_{k} \frac{\partial v_{i}}{\partial x_{k}}\right)=-\frac{\partial p}{\partial x_{i}}+\frac{\partial}{\partial x_{k}}\left\lfloor\mu\left(\frac{\partial v_{i}}{\partial x_{k}}\right)\right\rfloor .
$$

Уравнение энергии запишем в предположении локального термодинамического равновесия (когда в каждой точке можно определить температуру среды $T$ ), несжимаемости среды, отсутствия внешних сил и вязкой диссипации кинетической энергии [3]

$$
\rho\left(\frac{\partial c T}{\partial t}+\mathbf{v} \cdot \nabla c T\right)=-\nabla \cdot \mathbf{q}+h_{2} \nabla \cdot \mathbf{J}_{2}, \quad h_{2}=c_{2} T .
$$

Здесь $c$ - эффективная удельная теплоемкость дисперсной смеси, $h_{2}, c_{2}$ - удельная энтальпия и теплоемкость вещества дисперсной фазы, q - вектор потока энергии, обусловленного кондуктивным переносом тепла и диффузионным переносом наночастиц. Величина q может быть вычислена по формуле, предлагаемой в [3]:

$$
\mathbf{q}=-k \nabla T+h_{2} \mathbf{J}_{2}
$$

где $k-$ эффективная теплопроводность суспензии. Из уравнения (5) видно, что внутренняя энергия смеси может меняться как за счет кондуктивного потока тепла, так и из-за диффузионного переноса частиц. В уравнении (5) в отличие от уравнения энергии, приведенного в [3], учтено то обстоятельство, что эффективная теплоемкость наносуспензии, вообще говоря, зависит от объемной концентрации частиц (которая в свою очередь может зависеть от времени и координат), и в общем случае ее нельзя выносить за знак производной.

\section{2. Диффузионные потоки}

Относительное движение частиц в наносуспензии возникает под действием различных факторов, среди которых, как установлено в [3], наиболее существенными являются диффузия за счет броуновского движения (броуновская диффузия) и термодиффузия. Они возникают из-за наличия в смеси градиентов концентрации частиц и температуры и в первом приближении пропорциональны соответствующим градиентам. Полагается, что общий диффузионный поток дисперсной фазы складывается из броуновской диффузии и термодиффузии $[3,4]$

$$
\mathbf{J}_{2}=-\rho_{2}^{\circ} D_{B} \nabla \alpha_{2}-\rho_{2}^{\circ} D_{T} \frac{\nabla T}{T},
$$

где $D_{B}, D_{T}$ - коэффициенты броуновской диффузии и термодиффузии, которые могут быть вычислены по известным формулам $[11,12]$

$$
D_{B}=\frac{k_{B} T}{3 \pi \mu_{1} d_{2}}, \quad D_{T}=\beta \frac{\mu_{1} \alpha_{2}}{\rho_{1}^{\circ}}, \quad \beta=0.26 \frac{k_{1}}{2 k_{1}+k_{2}} .
$$

Здесь $k_{B}, d_{2}, \mu_{1}, k_{1}, k_{2}$ - постоянная Больцмана, диаметр частицы, динамическая вязкость жидкости, а также коэффициенты теплопроводности жидкости и вещества частиц соответственно. 


\section{3. Несжимаемость суспензии}

Выясним, когда выполняется условие $\nabla \cdot \mathbf{v}=0$, называемое условием несжимаемости суспензии. Для этого в уравнениях неразрывности (2) с помощью (1) перейдем к объемным концентрациям и сложим их, затем, учитывая соотношение $\mathbf{J}_{1}+\mathbf{J}_{2}=0$, получим уравнение

$$
\nabla \cdot \mathbf{v}=\left(\frac{1}{\rho_{1}^{\circ}}-\frac{1}{\rho_{2}^{\circ}}\right) \nabla \cdot \mathbf{J}_{2} .
$$

Отсюда видно, что условие несжимаемости суспензии $\nabla \cdot \mathbf{v}=0$ выполняется в двух случаях. В первом случае, когда $\rho_{1}^{\circ}=\rho_{2}^{\circ}$ (равноплотная суспензия $\left.(\mathrm{PC})\right)$, уравнение неразрывности для дисперсной фазы примет вид

$$
\frac{\partial \alpha_{2}}{\partial t}+\mathbf{v} \cdot \nabla \alpha_{2}=-\frac{1}{\rho_{2}^{\circ}} \nabla \cdot \mathbf{J}_{2} .
$$

Во втором случае, когда $\nabla \cdot \mathbf{J}_{2}=0$, имеем

$$
\frac{\partial \alpha_{2}}{\partial t}+\mathbf{v} \cdot \nabla \alpha_{2}=0
$$

Второй случай реализуется, в частности, в предельной ситуации, когда диффузионные потоки различного вида компенсируют друг друга, и результирующий диффузионный поток вещества в суспензии в целом отсутствует $-\mathbf{J}_{1}, \mathbf{J}_{2}=0$. На такое предельное состояние смеси указано в [9]. Это предельное состояние назовем условно „диффузионно-равновесной суспензией“ (ДРС). Рассмотрим его более подробно.

Для того чтобы было $\mathbf{J}_{2}=0$ (а значит, и $\mathbf{J}_{1}=0$ ), параметры $T, \alpha_{2}$, согласно (6), должны удовлетворять уравнению

$$
D_{B} \nabla \alpha_{2}+D_{T} \frac{\nabla T}{T}=0
$$

которое можно привести с учетом (7) к виду

$$
\begin{gathered}
\delta_{B} T \nabla \alpha_{2}+\delta_{T} \alpha_{2} \frac{\nabla T}{T}=0, \\
\delta_{B}=\frac{k_{B}}{3 \pi \mu_{1} d_{2}}, \quad \delta_{T}=\beta \frac{\mu_{1}}{\rho_{1}^{\circ}} .
\end{gathered}
$$

Деля обе части уравнения (9) на $\alpha_{2} T$ и объединяя слагаемые, придем к уравнению

$$
\nabla\left(\delta_{B} \ln \alpha_{2}-\delta_{T} \frac{1}{T}\right)=0,
$$

которое выполняется, если только

$$
\ln \alpha_{2}-\frac{\delta}{T}=\text { const, } \quad \delta=\frac{\delta_{T}}{\delta_{B}} .
$$

Это соотношение определяет связь между концентрацией и температурой, которая должна иметь место для отсутствия потока вещества. Постоянную в правой части (10) можно определить по характерным значениям $\alpha_{2}, T$ в некотором фиксированном, например

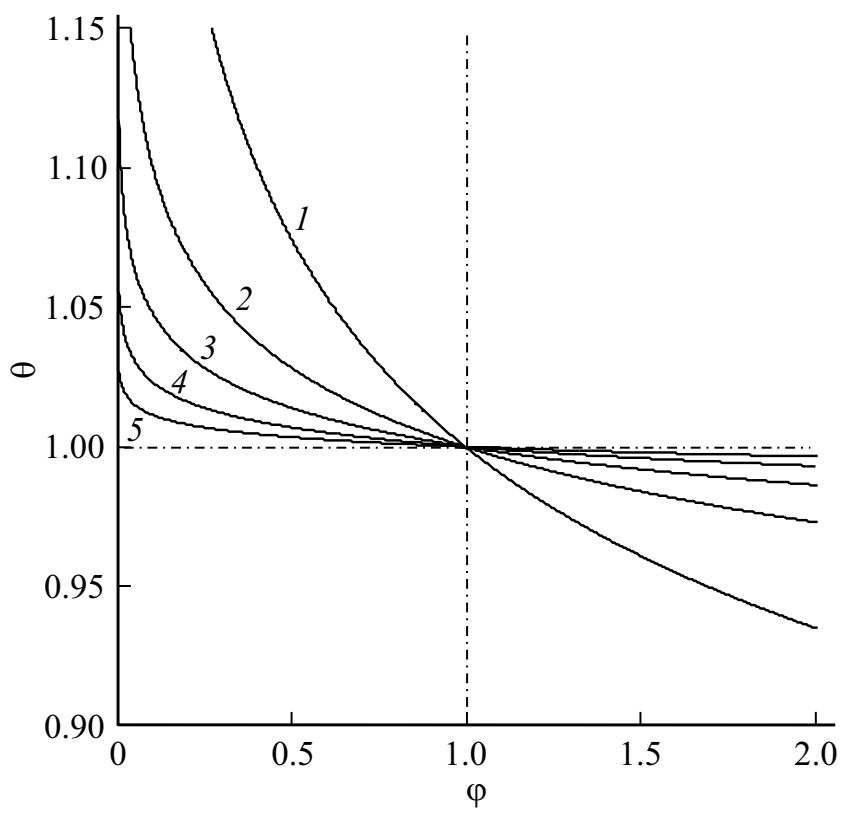

Рис. 1. Зависимость $\theta(\varphi)$ в случае ДРС при разных значениях $A=10$ (кривая 1), 25 (2), 50 (3), 100 (4), 200 (5).

на бесконечности, состоянии среды $\alpha_{2}=\alpha_{2 \infty}, T=T_{\infty}$. Соотношение (10) удобно переписать относительно этих характерных значений в форме

$$
\theta=\frac{A}{A+\ln \varphi}
$$

$$
\begin{aligned}
\varphi & =\frac{\alpha_{2}}{\alpha_{2 \infty}}, \quad \theta=\frac{T}{T_{\infty}}, \quad A=\frac{D_{T \infty}}{\alpha_{2 \infty} D_{B \infty}}=\beta \mathrm{Sc}, \\
\mathrm{Sc} & =\frac{\mu}{\rho_{1}^{\circ} D_{B \infty}}, \quad D_{B \infty}=\delta_{B} T_{\infty}, \quad D_{T \infty}=\delta_{T} \alpha_{2 \infty}
\end{aligned}
$$

или

$$
\varphi=\exp \left[A\left(\frac{1}{\theta}-1\right)\right],
$$

где $\mathrm{Sc}$ - число Шмидта. Из (11) следует, что значениям $\varphi<1$ соответствуют значения $\theta>1$ и, наоборот, при $\varphi>1$ получим $\theta<1$.

Поскольку параметр $A$ обычно принимает достаточно большие значения (см. ниже), то, как видно из представленных соотношений, малым изменениям температуры соответствуют большие изменения объемной концентрации частиц. Это обстоятельство позволяет пренебрегать влиянием температуры на эффективные теплофизические коэффициенты наножидкости, находящейся в состоянии ДРС, по сравнению с влиянием на них объемной концентрации дисперсной фазы.

Интересно, что при $\varphi<\varphi_{*}=\exp (-A)$ состояние ДРС отсутствует, так как при этих значениях параметра $\varphi$ температура становится отрицательной. Ввиду больших значений $A$ величина $\varphi_{*}$ достаточно мала, $\varphi_{*} \ll 1$. Очевидно, величина $\varphi$ не может превышать критического значения $\varphi_{* *}=\alpha_{2 *} / \alpha_{2 \infty} \geq 1$, где $\alpha_{2 *}$ - объемная доля дисперсной фазы при наиболее плотной упаковке частиц 
$\left(\alpha_{2 *} \cong 0.74\right)$. Таким образом, имеет место соотношение $\varphi_{*}<\varphi<\varphi_{* *}$.

Типичный вид зависимости (11) показан на рис. 1 при разных значениях параметра $A=10$ (кривая 1), 25 (2), 50 (3), 100 (4), 200 (5). Значение $A=100$ приблизительно соответствует, например, смеси воды и частиц алюминия диаметром $10 \mathrm{~nm}$ при характерном значении температуры $T_{\infty}=300 \mathrm{~K}$. Каждая из построенных кривых имеет свою асимптоту $\varphi=\varphi_{*}=\exp (-A)$. Штрихпунктирные линии $\varphi=1$ и $\theta=1$ служат асимптотами кривых рассматриваемой зависимости соответственно при неограниченном уменьшении и увеличении параметра $A$.

Важно иметь в виду, что из условия $\nabla \cdot \mathbf{v}=0$ еще не следует отсутствие диффузионных потоков компонентов смеси.

\section{4. Эффективные параметры смеси}

В рамках рассматриваемого подхода с диффузионными потоками влияние состава смеси непосредственно проявляется через физико-химические параметры, определяющие состояние смеси (плотность, коэффициенты вязкости, теплоемкости, теплопроводности смеси и т. п.). Приведем некоторые соотношения для этих параметров. Для плотности и теплоемкости смеси имеются универсальные соотношения

$$
\rho=\left(1-\alpha_{2}\right) \rho_{1}^{\circ}+\alpha_{2} \rho_{2}^{\circ}, \quad \rho c=\left(1-\alpha_{2}\right) c_{1} \rho_{1}^{\circ}+\alpha_{2} c_{2} \rho_{2}^{\circ},
$$

где $c_{1}, c_{2}$ - удельные теплоемкости жидкости и вещества частиц.

Для эффективных коэффициентов вязкости и теплопроводности существуют различные зависимости, среди которых необходимо отметить известные формулы Эйнштейна [9] и Максвелла [13]

$$
\begin{gathered}
\mu=\mu_{1}\left(1+2.5 \alpha_{2}\right), \\
k=k_{1}\left[\frac{k_{2}+2 k_{1}-2 \alpha_{2}\left(k_{1}-k_{2}\right)}{k_{2}+2 k_{1}+\alpha_{2}\left(k_{1}-k_{2}\right)}\right] .
\end{gathered}
$$

Эти формулы справедливы для малых объемных концентраций дисперсной фазы в наножидкости. При умеренных и больших объемных содержаниях частиц для расчета эффективной вязкости суспензии целесообразно использовать формулу Муни [14], которая довольно хорошо описывает многочисленные экспериментальные данные в широком диапазоне объемных содержаний дисперсной фазы $\alpha_{2}$ [15]

$$
\mu=\mu_{1} \exp \left(\frac{2.5 \alpha_{2}}{1-\alpha_{2} / \alpha_{2 *}}\right) .
$$

Здесь $\alpha_{2 *}$ - объемная доля дисперсной фазы при наиболее плотной упаковке частиц $\left(\alpha_{2 *} \cong 0.74\right)$. Коэффициент 2.5 обеспечивает переход формулы (16) к формуле Эйнштейна (14) при малых объемных долях дисперсной фазы. Формулы (14)-(16) в настоящее время широко применяются при расчетах течений наножидкостей.

Для описания эффективной теплопроводности наносуспензий при умеренных и больших концентрациях частиц имеются эмпирические формулы, некоторые из которых приведены в $[3,16]$. В последнее время большое внимание уделяется моделированию и расчету эффективных коэффициентов вязкости и теплопроводности наносуспензий с привлечением методов молекулярной динамики [17-19].

Соотношения (13)-(16) характеризуют зависимость эффективных теплофизических параметров смеси от объемной концентрации дисперсной фазы и могут быть использованы для описания состояния наносуспензии. Отметим, что при не очень больших изменениях температуры теплофизические параметры отдельных составляющих дисперсной смеси можно считать постоянными.

Далее рассмотрим течение наножидкости в пограничном слое при выполнении условия несжимаемости суспензии $\nabla \cdot \mathbf{v}=0$.

\section{5. Приближение пограничного слоя и постановка задачи}

\section{1. Уравнения пограничного слоя и граничные условия}

Рассмотрим стационарное движение несжимаемой наножидкости в пограничном слое на продольно обтекаемой плоской пластине. Уравнение несжимаемости суспензии $(\nabla \cdot \mathbf{v}=0)$, а также уравнения количества движения (4) и энергии (5) в декартовой системе координат в приближении пограничного слоя примут вид (ось $x$ направлена вдоль пластины, а ось $y-$ поперек неe)

$$
\begin{gathered}
\frac{\partial u}{\partial x}+\frac{\partial v}{\partial y}=0, \quad \rho\left(u \frac{\partial u}{\partial x}+v \frac{\partial u}{\partial y}\right)=\frac{\partial}{\partial y}\left(\mu \frac{\partial u}{\partial y}\right), \\
\rho\left(u \frac{\partial c T}{\partial x}+v \frac{\partial c T}{\partial y}\right)=\frac{\partial}{\partial y}\left(k \frac{\partial T}{\partial y}\right) \\
+\rho_{2}^{\circ} c_{2}\left\lfloor D_{B} \frac{\partial \alpha_{2}}{\partial y} \frac{\partial T}{\partial y}+\frac{D_{T}}{T}\left(\frac{\partial T}{\partial y}\right)^{2}\right\rfloor .
\end{gathered}
$$

Сюда необходимо добавить уравнения диффузии наночастиц, соответствующие рассмотренным выше случаям, приводящим к несжимаемости суспензии. Например, в случае ДРС уравнение диффузии в приближении пограничного слоя запишется в форме

$$
D_{B} \frac{\partial \alpha_{2}}{\partial y}+\frac{D_{T}}{T} \frac{\partial T}{\partial y}=0 .
$$

При этом слагаемое, содержащее квадратные скобки в последнем уравнении системы (17) обращается в нуль.

Аналогично запишутся уравнения диффузии для других случаев. 
Поставим граничные условия. На пластине для жидкости ставится условие прилипания, а также задается постоянная температура $T_{w}$. На бесконечности выполняются условия набегающего потока. Так что, имеем

$$
\begin{gathered}
y=0: \quad u=v=0, \quad T=T_{w}, \\
y=\infty: \quad u=u_{\infty}, \quad \alpha_{2}=\alpha_{2 \infty}, \quad T=T_{\infty} .
\end{gathered}
$$

Заметим, что в случае ДРС вместо уравнения (18) можно использовать найденное выше соотношение (12). Тогда условие на бесконечности для $\alpha_{2}$ отпадает.

\section{2. Постановка задачи в автомодельных переменных}

Введем, как обычно, функцию тока $\psi$ согласно равенствам

$$
u=\frac{\partial \psi}{\partial y}, \quad v=-\frac{\partial \psi}{\partial x} .
$$

Тогда первое уравнение в (17) удовлетворяется тождественно. Перейдем к автомодельным переменным [10]

$$
\eta=y \sqrt{\frac{u_{\infty}}{\mathrm{v}_{1} x}}, \quad f(\eta)=\frac{\psi}{\sqrt{\mathrm{v}_{1} u_{\infty} x}}, \quad \mathrm{v}_{1}=\frac{\mu_{1}}{\rho_{1}^{\circ}} .
$$

В новых переменных уравнения количества движения и энергии (17) примут вид

$$
\begin{gathered}
M f^{\prime \prime \prime}+\dot{M} \alpha_{2}^{\prime} f^{\prime \prime}+\frac{R}{2} f f^{\prime \prime}=0, \\
K \theta^{\prime \prime}+\frac{\operatorname{Pr}}{2}\left(C \theta^{\prime}+\dot{C} \theta \alpha_{2}^{\prime}\right) f \\
+\left(\dot{K}+\frac{\theta}{\mathrm{Le}}\right) \alpha_{2}^{\prime} \theta^{\prime}+\frac{B}{\mathrm{Le}} \frac{\alpha_{2}}{\theta} \theta^{\prime 2}=0, \\
R=\frac{\rho}{\rho_{1}^{\circ}}, \quad M=\frac{\mu}{\mu_{1}}, \quad C=\frac{\rho c}{\rho_{1}^{\circ} c_{1}}, \\
k \frac{k}{k_{1}}, \quad \operatorname{Pr}=\frac{c_{1} \mu_{1}}{k_{1}}, \quad \operatorname{Le}=\frac{k_{1}}{\rho_{2}^{\circ} c_{2} D_{B \infty}}, \\
B=\frac{D_{T \infty}}{D_{B \infty}}=\alpha_{2 \infty} A=\alpha_{2 \infty} \beta \mathrm{Sc}, \\
\dot{M}=\frac{d M}{d \alpha_{2}}, \quad \dot{C}=\frac{d C}{d \alpha_{2}}, \quad \dot{K}=\frac{d K}{d \alpha_{2}},
\end{gathered}
$$

где штрих означает производную по автомодельной переменной $\eta$, а точка наверху - производную по $\alpha_{2}$, обозначения $\mathrm{Pr}$, Le представляют собой числа Прандтля и Льюиса соответственно. Первое уравнение системы (20) в случае отсутствия в смеси дисперсной фазы $(R=1$, $M=1, \dot{M}=0)$ переходит в частный случай уравнения Фолкнера-Скэн, описывающий течение жидкости (без частиц) в пограничном слое на плоской пластине [10].

В новых переменных граничные условия (19) запишутся как

$$
\begin{gathered}
\eta=0: \quad f=0, \quad f^{\prime}=0, \quad \theta=\theta_{w}, \\
\eta=\infty: \quad f^{\prime}=1, \quad \alpha_{2}=\alpha_{2 \infty}, \theta=1 .
\end{gathered}
$$

К системе (20) необходимо добавить уравнение диффузии в автомодельных переменных, соответствующее разным случаям выполнения условия несжимаемости суспензии. Например, в случае ДРС, используя вытекающее из (12) соотношение

$$
\alpha_{2}^{\prime}=\xi(\theta) \theta^{\prime}, \quad \xi(\theta)=-\frac{B}{\theta^{2}} \exp \left[A\left(\frac{1}{\theta}-1\right)\right]
$$

и уравнение диффузии (18), систему (20) можно переписать в более компактной форме

$$
\begin{gathered}
M f^{\prime \prime \prime}+\dot{M} \xi \theta^{\prime} f^{\prime \prime}+\frac{R}{2} f f^{\prime \prime}=0, \\
K \theta^{\prime \prime}+\dot{K} \xi \theta^{\prime 2}+\frac{\operatorname{Pr}}{2}(C+\dot{C} \theta \xi) \theta^{\prime} f=0 .
\end{gathered}
$$

Коэффициенты $R, M, C, K, \dot{M}, \dot{K}, \dot{C}$, зависящие от $\alpha_{2}$, с помощью (12) сводятся к зависимостям от $\theta$. Таким образом, система (22) фактически представляет собой систему дифференциальных уравнений для двух неизвестных функций $f$ и $\theta$.

В более общем случае $\nabla \cdot \mathbf{J}_{2}=0$, приводящем также к несжимаемости суспензии, как показал анализ, течение смеси в пограничном слое описывается системой (22), соответствующей ДРС.

Уравнение диффузии (8), отвечающее РС в автомодельных переменных, примет вид

$$
\frac{\mathrm{Sc}}{2} f \alpha_{2}^{\prime}+\left(\alpha_{2}^{\prime} \theta+A \alpha_{2} \frac{\theta^{\prime}}{\theta}\right)^{\prime}=0, \quad A=\beta \mathrm{Sc} .
$$

Это уравнение нужно присоединить к системе (20) в случае РС.

\section{6. Некоторые результаты расчетов}

Проанализируем результаты расчетов в случаях ДРС и РС, полученные численным методом Рунге-Кутта [20] после приведения краевой задачи к задаче Коши методом преобразования [21]. В случае ДРС интегрировалась система уравнений (22), а в случае РС - система (20), (23) с граничными условиями (21), где для ДРС условие для объемной концентрации на бесконечности должно быть исключено. Расчеты проводились для воды с частицами алюминия при температуре набегающего потока $T_{\infty}=300 \mathrm{~K}$ и постоянной температуре пластины $T_{w}=$ const. Рассматривались случаи, когда температура пластины больше температуры набегающей жидкости $T_{w}>T_{\infty}$ и когда, наоборот, $T_{w}<T_{\infty}$. Ниже (рис. $2-5$ ) показаны распределения безразмерной продольной скорости $U=u / u_{\infty}=f^{\prime}$, относительной объемной концентрации частиц $\varphi=\alpha_{2} / \alpha_{2 \infty}$ и безразмерной температуры $\theta=T / T_{\infty}$ в зависимости от автомодельной переменной $\eta$. Штриховые кривые отвечают „чистой“ (без частиц) жидкости.

На рис. 2 представлены результаты расчета для ДРС при диаметре частиц $d_{2}=1.25 \mathrm{~nm}$, объемном содержании дисперсной фазы в набегающем потоке $\alpha_{2 \infty}=0.05$ 


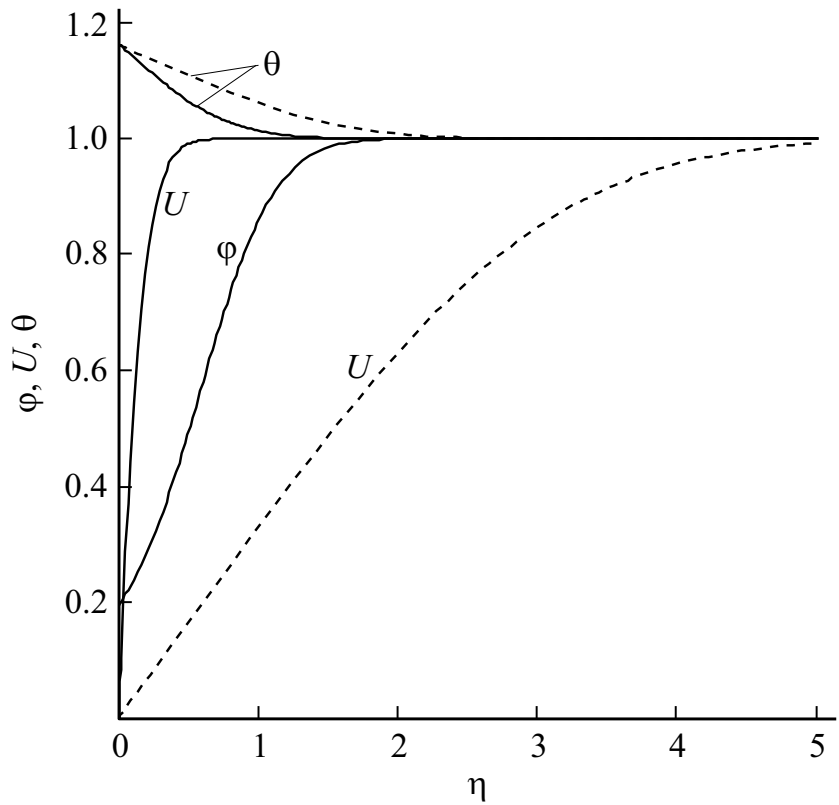

Рис. 2. Распределение безразмерных параметров ДРС в пограничном слое в зависимости от автомодельной переменной $\eta$ при $T_{w}>T_{\infty}$.

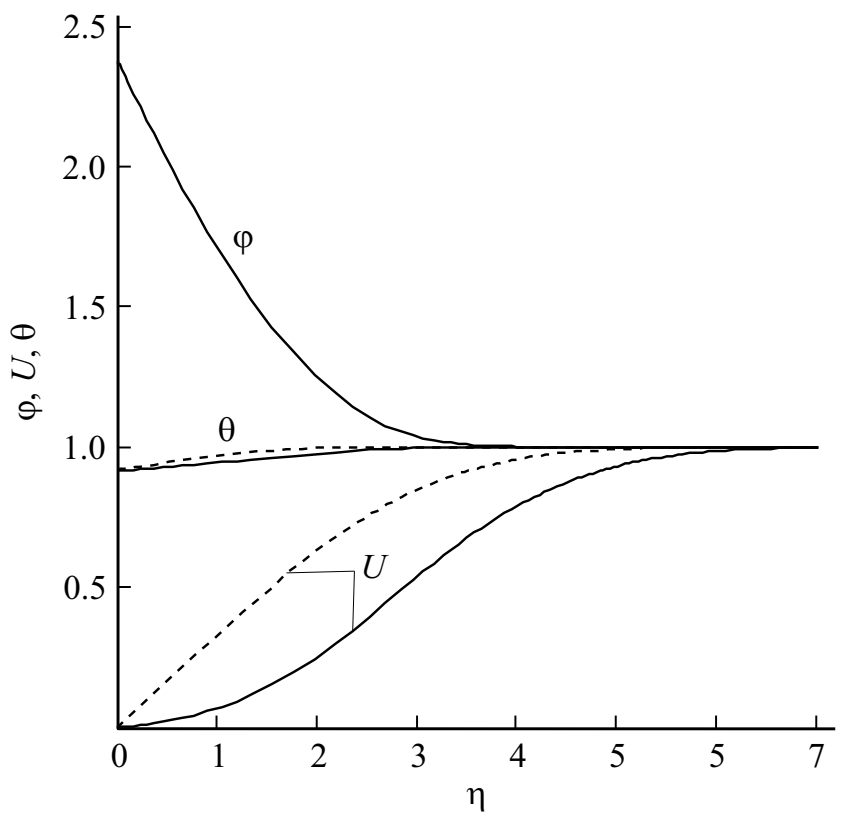

Рис. 3. Распределение безразмерных параметров ДРС в пограничном слое в зависимости от автомодельной переменной $\eta$ при $T_{w}<T_{\infty}$.

в случае $T_{w}>T_{\infty}$. Видно, что наличие дисперсных частиц в потоке приводит к существенному уменьшению характерной толщины пограничного слоя для скорости. Температура жидкости при наличии частиц заметно меньше, чем при их отсутствии.

Рис. 3 соответствует температурному режиму $T_{w}<T_{\infty}$ при $d_{2}=1 \mathrm{~nm}, \alpha_{2 \infty}=0.1$. В этом случае величины $\varphi, \theta$ ведут себя по сравнению с предыдущим случаем совсем по-другому: с ростом $\eta$ объемная концентрация частиц $\varphi$ уменьшается, а температура $\theta$ увеличивается, тогда как при $T_{w}>T_{\infty}$ (рис. 2) параметр $\varphi$ увеличивается, а $\theta$ уменьшается. Отметим, что скорость суспензии $U$ в пограничном слое в отличие от предыдущего случая меньше, чем в „чистой“ жидкости. Причем кривая $U$ в рассматриваемом варианте имеет

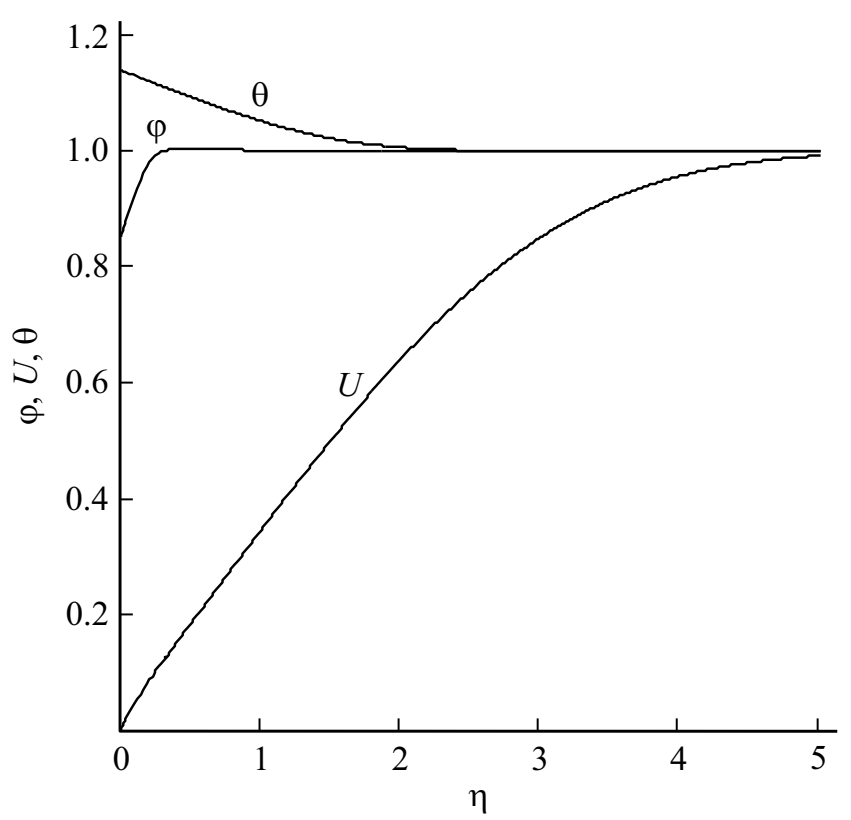

Рис. 4. Распределение безразмерных параметров РС в пограничном слое в зависимости от автомодельной переменной $\eta$ при $T_{w}>T_{\infty}$.

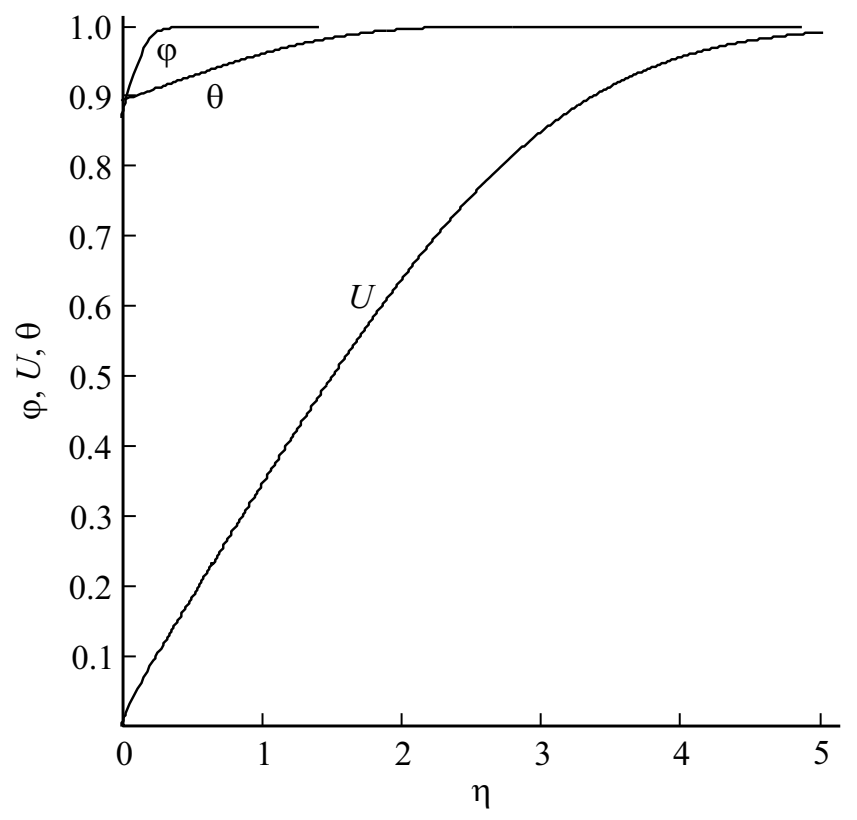

Рис. 5. Распределение безразмерных параметров РС в пограничном слое в зависимости от автомодельной переменной $\eta$ при $T_{w}<T_{\infty}$. 
точку перегиба. Данное обстоятельство принципиально влияет на вопросы устойчивости движения в пограничном слое. Однако обсуждение этого аспекта выходит за рамки настоящей работы (подробности см. в [9]).

Заметим, что значения температуры и концентрации частиц на рис. 2,3 удовлетворяют аналитической зависимости, полученной для ДРС (11). Это подтверждает корректность проведенных расчетов.

На рис. 4 представлены распределения параметров РС при $d_{2}=1.16 \mathrm{~nm}, \alpha_{2 \infty}=0.012$ и $T_{w}>T_{\infty}$. Интересно, что продольная скорость РС, несмотря на наличие диффузионных потоков вещества, ведет себя точно так же, как при обтекании пластины „чистой“ жидкостью. Видно, что в случае РС концентрационный пограничный слой заметно тоньше, чем пограничные слои других параметров. Причем значение объемной концентрации частиц на поверхности пластины существенно выше, чем в случае ДРС (рис. 2). Это связано с тем, что в данном примере расчета с РС имеет место диффузионный поток частиц, направленный в сторону стенки и приводящий к росту концентрации частиц вблизи нее.

Рис. 5 иллюстрирует поведение РС в пограничном слое в случае $T_{w}<T_{\infty}$ при $d_{2}=1 \mathrm{~nm}, \alpha_{2 \infty}=0.012$. Отметим, что в данном случае все три параметра $\varphi, \theta, U$ с ростом автомодельной переменной $\eta$ увеличиваются.

\section{Заключение}

Изучено поведение наножидкости в пограничном слое на продольно обтекаемой пластине в рамках диффузионного приближения. Сформулирована система уравнений, описывающих динамику наносуспензии при наличии диффузионных потоков. Получена связь между температурой жидкости и концентрацией дисперсной фазы в состоянии диффузионного равновесия. Проведены расчеты параметров пограничного слоя при разных температурных режимах, а именно в случаях, когда температура пластины больше или меньше температуры набегающего потока наножидкости. Обнаружено кардинально разное распределение параметров наножидкости в пограничном слое в рассматриваемых случаях. Причем скорость смеси в пограничном слое может оказаться в зависимости от температурного режима как меньше, так и больше, чем в случае обтекания пластины жидкостью без частиц.

Отметим, что в общем случае, когда суспензия не является несжимаемой, система уравнений пограничного слоя будет иметь более сложный вид. При этом возникает проблема конкретизации второй вязкости для смеси. Задача о пограничном слое в наножидкости в такой постановке требует отдельного рассмотрения.

Результаты и выводы настоящей работы могут быть полезны для оценки и проверки результатов, полученных на основе более сложных моделей.
Работа выполнена при финансовой поддержке Министерства образования и науки Республики Казахстан (грант № 3486/ГФ4).

\section{Список литературы}

[1] Choi U.S. // Developments and Applications of NonNewtonian Flows. Eds. D.A. Siginer, H.P. Wang. FED-V. 231/MD-Vol. 66. NY.: ASME, 1995. P. 99-105.

[2] Бардаханов С.П., Новопашин С.А., Серебрякова М.А. // Наносистемы: физ., хим., математика. 2012. Т. 3. № 1. C. 27-33.

[3] Buongiorno J. // J. Heat Transfer. 2006. Vol. 128. P. 240-250.

[4] Kuznetsov A.V., Nield D. // Int. J. Thermal Sci. 2010. Vol. 49. P. 243-247.

[5] Kowsary F., Heyhat M. // Nanomech. Sci. Technology: An Inter. J. 2010. Vol. 1. N 3. P. 257-272.

[6] Yasin M.H., Ishak A., Pop I. // J. Porous Media. 2016. Vol. 19. N 4. P. 331-338.

[7] Седов Л.И. Механика сплошной среды. Т. 1. М.: Наука, 1983. 528 c. [Sedov L.I. Mechanics of Continuous Media. World Scientific, 1997.]

[8] Нигматулин Р.И. Динамика многофазных сред. Т. 1. М.: Наука, 1987. 464 c. [Nigmatulin R.I. Dynamics of Multiphase Media. NY: CRC Press, 1990.]

[9] Ландау Л.Д., Лифиии Е.M. Теоретическая физика. T. VI. Гидродинамика. М.: Наука, 1988. 736 с. [Landau L.D., Lifshitz E.M. Fluid Mechanics. NY.: Pergamon Press, 1987.]

[10] Лойцянский Л.Г. Механика жидкости и газа. М.: Наука, 1987. $840 \mathrm{c}$.

[11] Einstein A. Investigations on the Theory of Brownian Movement. NY.: Dover, 1954. 132 p.

[12] McNab G.S., Meisen A. // J. Colloid Interface Sci. 1973. Vol. 44. N 2. P. 339-351.

[13] Maxwell J.C. A treatise on electricity and magnetism. Oxford: Clarendon Press, 1881. 481 p.

[14] Mooney M. // J. Colloid Sci. 1951. Vol. 6. N 2. P. 162-175.

[15] Smith T.L., Bruce C.A. // J. Colloid Interface Sci. 1979. Vol. 72. N 1. P. 13-26.

[16] Pak B.C., Cho Y. // Exp. Heat Transfer. 1998. Vol. 11. P. $151-170$.

[17] Рудяк В.Я., Белкин А.А., Егоров В.В. // ЖТФ. 2009. Т. 79. Вып. 8. C. 18-25. [Rudyak V.Ya., Belkin A.A., Egorov V.V. // Technical Physics. 2009. Vol. 54. N 8. P. 1102-1109.]

[18] Рудяк В.Я., Краснолуцкий С.Л. // ЖТФ. 2015. Т. 85. Вып. 6. C. 9-16. [Rudyak V.Ya., Krasnolutskii S.L. // Technical Physics. 2015 Vol. 60. N 6. P. 798-805.]

[19] Рудяк В.Я., Краснолуцкий С.Л. // ЖТФ. 2017. Т. 87. Вып. 10. C. 1450-1458. [Rudyak V.Ya., Krasnolutskii S.L. // Technical Physics. 2017. Vol. 62. N 10. P. 1456-1465.]

[20] Амосов А.А., Дубинский Ю.А., Копченова Н.В. Вычислительные методы для инженеров. М.: Высшая школа, 1994. $544 \mathrm{c}$

[21] Na T.Y. Computational methods in engineering boundary value problems. Academic Press, 1979. 295 p. 